$4^{0}$ Que l'actinisation des laits crus contrôlés doit donner satisfaction aux hygiénistes les plus rigoureux.

$5^{\circ}$ Que l'irradiation se faisant en un temps réduit, moins de $1 / 10^{e}$ de seconde, les accidents rencontrés avee les produits irradiés longuement (plusieurs minutes) ne doivent pas exister ici.

$6^{\circ}$ Que le lait actinisé doit être un aliment de choix pour les femmes enceintes ou nourrices : qualité qui doit se transmettre aux nourrissons.

\title{
C. Scientifiquement.
}

10 Que de la gélose irradiée, même au travers du verre, devient stérile en surface, pour un temps proportionnel à l'exposition aux rayons ultra-violets.

$2^{\circ}$ Que des colonies microbiennes sont tuées même à travers le verre des tubes à essais.

$3^{\circ}$ Que des modifications chimiques et physiques sont apportées dans les liquides actinisés.

$4^{\circ}$ Que de nombreuses expériences sont à faire tant au point de vue bactériologique, ehimique, physique, histochimique, etc., grâce à l'actinisateur W. P. de Stoutz.

$5^{\circ}$ Que, de même que la pasteurisation n'est pas la stérilisation, l'actinisation n'est pas l'irradiation.

\section{REVUE}

\section{LA CONSERVATION DES PRODUITS LAITIERS PAR LE PROCÉDÉ HOFIUS}

\author{
par G. GENIN
}

Ingénieur ehimiste E. P. C.

Les milieux intéressés portent actuellement beaucoup d'attention à un nouveau procédé de conservation du lait : le procédé Hofius, qui a fait l'objet, récemment, d'une importante étude du Professeur ScHWarz, du Preussischer Versuchs und Forschungsanstalt für Milchwirtschaft, publiée dans la revue allemande "Angewandte Chemie ".

\section{Description du procédé.}

D'après le Professeur Schwarz, le procédé consiste tout d'abord à chasser du lait les gaz qu'il peut contenir ; on le conserve ensuite dans une atmosphère d'oxygène sous une pression d'au moins 8 kilogrammes. Une pasteurisation préalable du lait n'est pas nécessaire. 
L'appareillage nécessaire à la conservation, tel qu'il a été imaginé par Th. Hofius, comprend un récipient cylindrique en acier V 2 A de $1 \mathrm{~mm}$. 5 d'épaisseur. Ce récipient est renforcé par deux frettes, soudées électriquement, et il est muni de deux robinets à vis, l'un commandant l'introduction du gaz, l'autre le remplissage ou le vidage du récipient.

Le lait, refroidi à au moins $8^{\circ} \mathrm{C}$, est introduit dans le récipient, de telle sorte qu'il soit au plus aux trois quarts plein. On introduit alors l'oxygène, au moyen d'un détendeur, de telle sorte que la pression à l'intérieur du cylindre atteigne 8 atmosphères. Au bout d'une minute, on ferme le robinet d'introduction du gaz et on agite vigoureusement le récipient pendant 2 à 3 minutes, puis laisse reposer pendant une minute. On ouvre alors la valve et on permet à l'oxygène mélangé à d'autres gaz, qui étaient contenus dans le lait, de s'échapper, en prenant bien soin qu'il ne pénètre pas d'air dans l'appareil. On remplit à nouveau d'oxygène sous une pression de 8 atmosphères, et on place l'appareil dans une chambre où la température ne dépasse pas $8^{\circ} \mathrm{C}$. en l'agitant tous les deux jours. Si la pression s'abaisse à l'intérieur du eylindre au-dessous de 7 atmosphères, on doit faire le plein avec de l'oxygène frais.

\section{Importance du contrôle de la température.}

Dans les essais effectués à Kiel, les récipients employés pour le stockage du lait étaient nettoyés par une solution de carbonate de soude chaude, puis traités par de la vapeur surchauffée et finalement lavés à l'eau froide. On les plaçait dans des chambres froides, dont la température était automatiquement réglée entre 3 et $6^{\circ} \mathrm{C}$.

Des essais ont été entrepris afin de bien mettre en évidence l'importance du facteur température. On a constaté que dans les chambres froides, le lait peut être conservé pendant une période de 14 jours sans que son acidité s'élève. Mais si la température atteint 18 à $22^{\circ} \mathrm{C}$. on constate une augmentation nette de l'acidité du lait au bout de 3 jours.

Le lait entier non traité, comme le lait entier qui a été rapidement chauffé, mais non pasteurisé, ont leur goût inchangé, lorsqu'ils sont conservés suivant les indications de Horius pendant 17 jours. Au bout de 28 jours, les résultats sont encore satisfaisants, mais le lait acquiert un très léger goût et une faible odeur de caramel. Au bout de 42 jours de stockage, le goût et l'odeur sont considérablement accentués, plus encore dans le cas du lait qui, avant le traitement, a été chauffé.

La conclusion de cette étude est que le stockage ne doit pas être prolongé au delà de 4 semaines.

Avec le petit-lait, la durée maximum possible de stockage est de 
18 jours, au bout de 28 jours en effet il apparaît avec ce lait un goût très net qui après 35 jours devient très désagréable. Le beurre conservé par le procédé Hofius acquiert dans tous les cas un goût malté.

Des essais sont en cours afin d'établir si l'efficacité du procédé est due à la formation d'eau oxygénée ; jusqu'ici il n'a pas été possible de déceler dans le lait la présence de peroxyde.

\section{BIBLIOGRAPHIE ANALYTIQUE}

\section{LES LIVRES}

Pijanowski (F.). - Etudes sur l'acidité du beurre. II. Nouvelle méthode de détermination de l'acidité du plasma du beurre, et comparaison de cette méthode avec les méthodes antérieures. Travaux et comptes rendus de l'Institut de Bactériologie et d'Industrie agricole de l'Ecole centrale agronomique de Varsovie et de l'Institut des Industries de Fermentation et de Bactériologie agricole du Musée d'Industrie et d'Agriculture de Varsovie, Varsovie, XXII, 1936, p. 189-204.

Les méthodes aetuelles de détermination de l'acidité du plasma du beurre étant d'exécution assez compliquée, et ne convenant pas très bien dans la pratique journalière, P. a imaginé une nouvelle méthode pouvant se résumer comme suit : 20 grammes de beurre sont pesés dans un tube à essai à fond plat et à parois épaisses de $23 \mathrm{~mm} . \times 150 \mathrm{~mm}$; puis le tube est plongé dans un bain-marie à $37^{\circ}-40^{\circ} \mathrm{C}$, pendant $15-20$ minutes. La température est ensuite élevée de $45-50^{\circ} \mathrm{C}$., puis elle est maintenue pendant 5 minutes. Le tube est ensuite bouché, puis centrifugé à 1.000 tours par minute, et plongé à nouveau dans le bain-marie à $45^{\circ}-50^{\circ} \mathrm{C}$. pendant plusieurs minutes. La couche de matière grasse est enlevée par décantation, et on ajoute au plasma brut restant quelques gouttes de phénolphtaléine à $2 \%$. Ensuite, titration jusqu'à obtention d'une couleur rose avee $\mathrm{n} / 10 \mathrm{Na}$ OH. Il est nécessaire de connaître la teneur en matière grasse (f) du beurre. L'acidité du plasma (K2) exprimée en centimètres cubes normaux par 100 grammes de plasma est obtenue par la formule suivante :

$$
\mathrm{K}^{2}=\frac{50 \mathrm{n}}{100-\mathrm{f}}
$$

'dans laquelle $\mathrm{n}$ est le nombre de centimètres cubes de $\mathrm{n} / 10 \mathrm{Na} \mathrm{OH}$ nécessaires pour neutraliser le plasma de 20 grammes de beurre. Si on utilise $\mathrm{m}$ grammes de beurre, au lieu de 20 grammes, la formule devient:

$$
\mathrm{K}^{2}=\frac{1.000 \mathrm{n}}{\mathrm{m}(100-\mathrm{f})}
$$

Pour une détermination pratique, on peut utiliser la formule :

$$
\mathrm{K}^{2}=\frac{1.000 \mathrm{n}}{\mathrm{m}(\mathrm{w}+\mathrm{s}+1)}
$$

dans laquelle w est le pourcentage d'eau et s celui de sel du beurre

C. WoLF. 